\title{
THE FUTURE OF PLANETARY ATMOSPHERIC, SURFACE, AND INTERIOR SCIENCE USING RADIO AND LASER LINKS
}

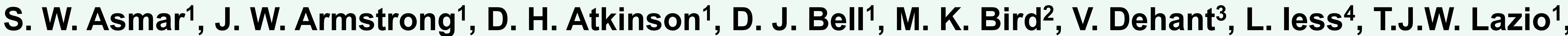 \\ I. R. Linscott ${ }^{5}$, A. J. Mannucci ${ }^{1}$, E. Mazarico ${ }^{6}$, R. S. Park1 ${ }^{1}$, M. Pätzold7, R. A. Preston ${ }^{1}$, \& R. A. Simpson ${ }^{5}$
}

\section{Introduction}

Studies of planetary systems using spacecraft radio links constitute the field of Radio Science (RS). RS experiments have been conducted on almost every planetary mission in the past five decades and have led to numerous discoveries.

With substantial technical advancements in recent years, the following significant accomplishments fit NASA's Planetary Vision 2050 themes:

ORIGINS:

-Elucidated the thermal history of the Moon from the GRAIL high precision gravitational field,

- Unveiled the interiors of Titan, Enceladus, Mercury, Phobos, Vesta, Ceres, and cometary nuclei from gravity fields, contributing to understanding their origins (Fig. 1), -Sounded Titan, Saturn, and Pluto's atmospheres, -Explored the surface properties of Pluto and 67P/Churyumov-Gerasimenko, and -Refined models for the atmospheres, surfaces, and interior structure of Mars and Venus.

- In progress: Juno and Cassini RS experiments are measuring the gravitational fields of Jupiter and Saturn to reveal their interior structures.

LIFE:

-Provided key evidence for identifying subsurface oceans on icy moons, helping expand our understanding of potentially habitable bodies.

\section{WORKINGS}

-Investigated the solar corona and the interaction of the solar wind with planetary atmospheres, and -Profiled the structure of Saturn's rings, which interact with moonlets.

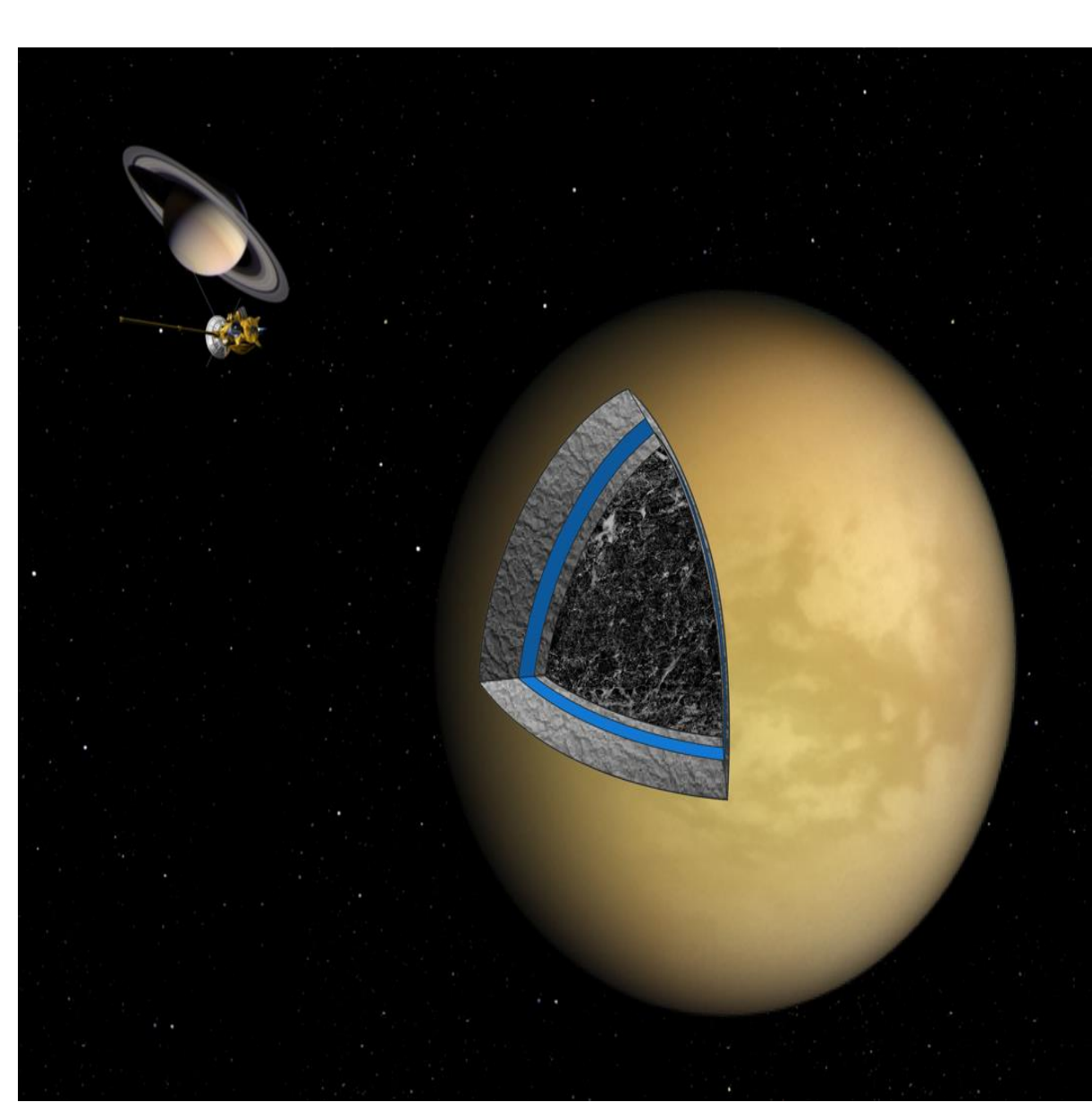

Fig. 1: Representation of Cassin Titan observations for deducing interior structure [1]

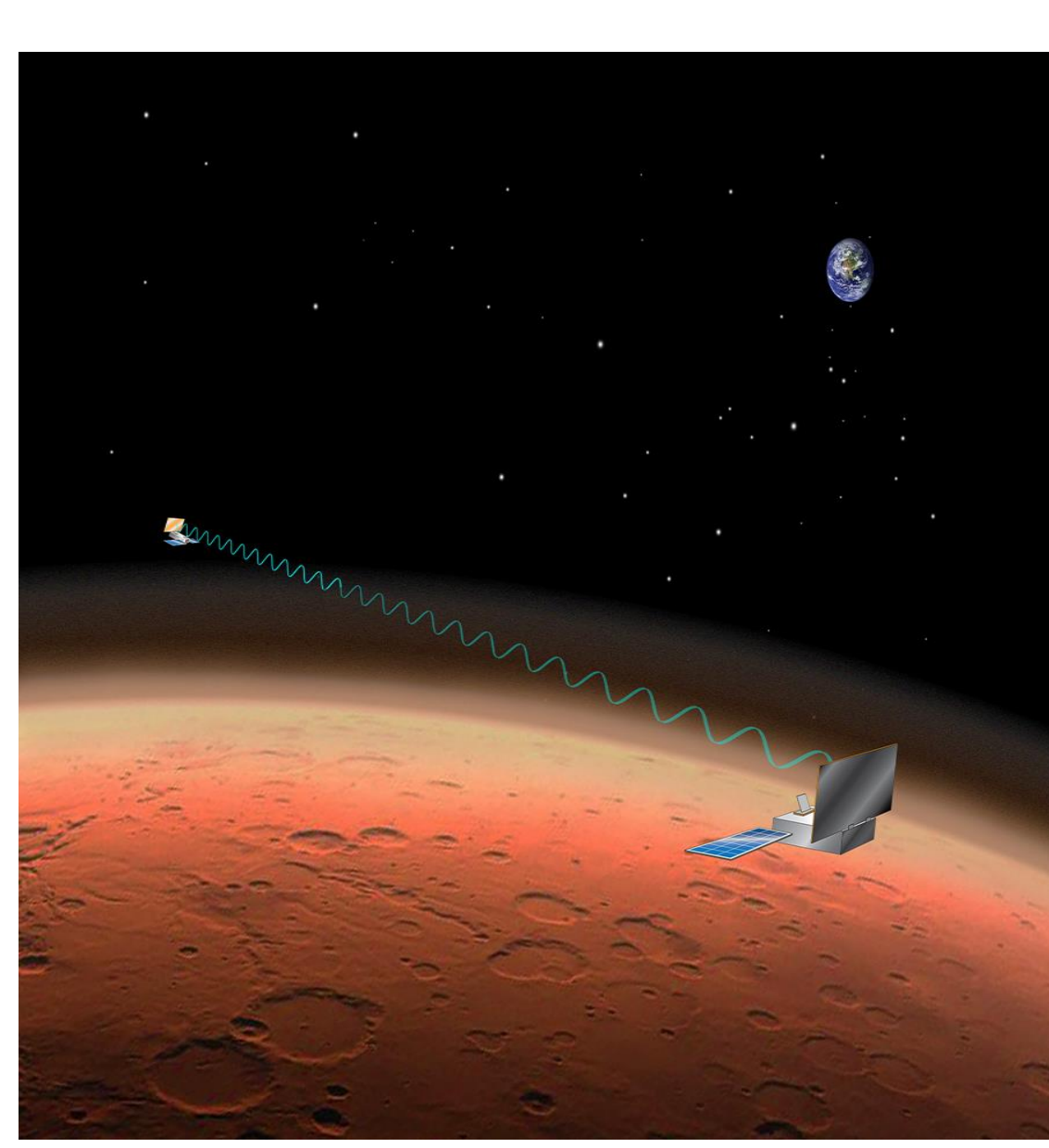

Fig 2: Artist conception of CubeSats Mars network for atmospheric occultations [?

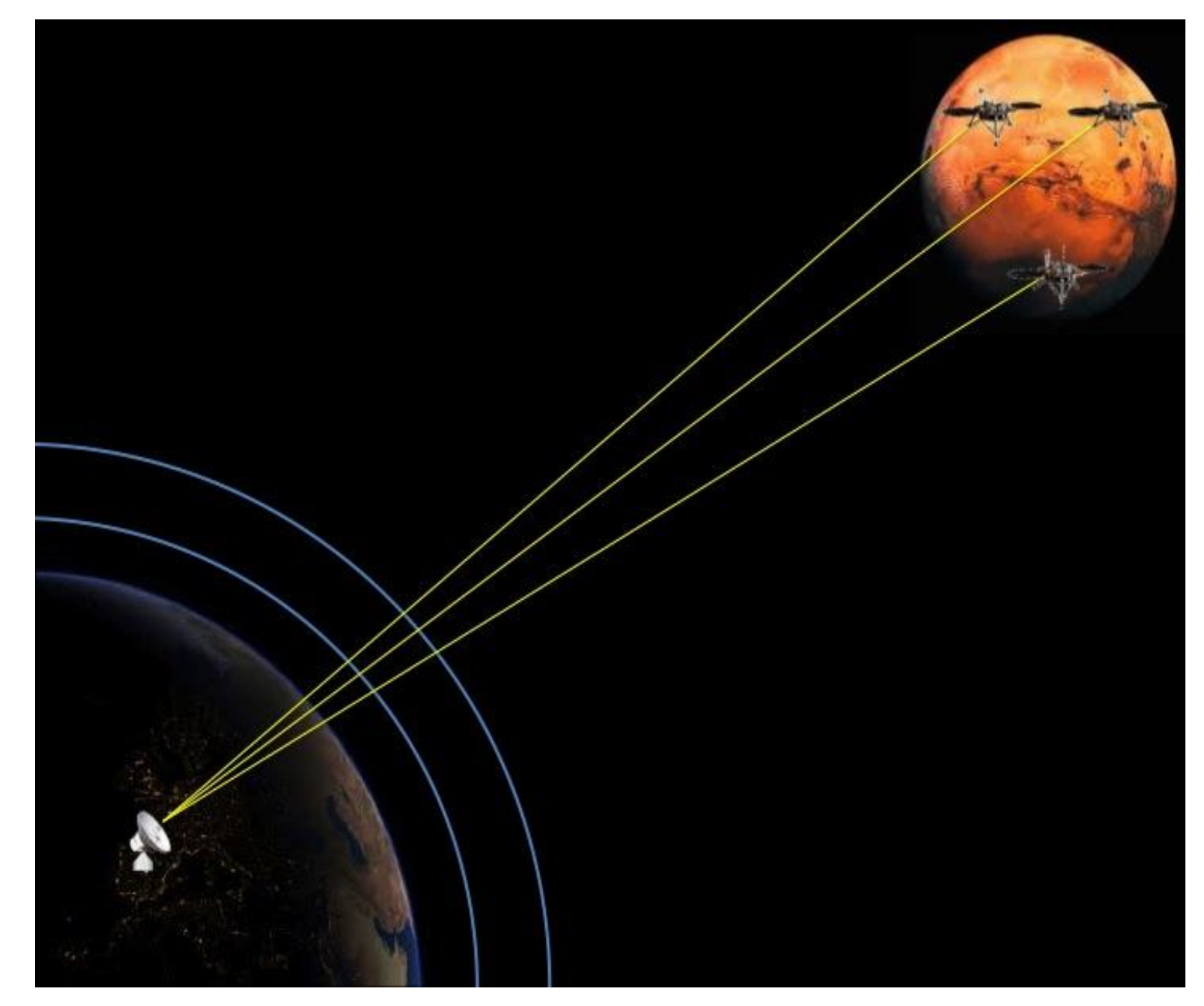

Fig. 3: Artist

conception of

interferometry

technique for

planetary

rotational/tidal

deformations [3]

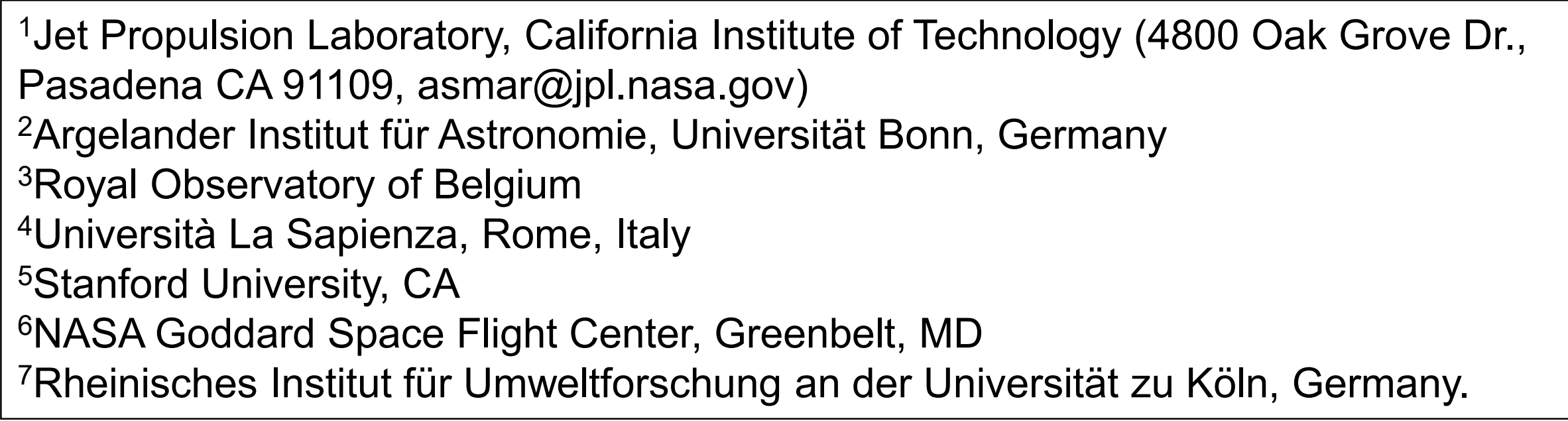

\section{Outlook to 2050}

InSight will soon characterize the Martian core and Akatsuki will study Venus' atmosphere via RS techniques. Experiments at Mercury, Jupiter, and other environments are in the development or planning phases. Over the next 30 years, advances in radio and laser technologies, such as ones shown below, could enable many scientific breakthroughs. With an order-of-magnitude improvement achieved in range-rate and similar improvement achievable in range accuracy, many discoveries akin to the unanticipated detection of buried empty lunar lava tubes with GRAIL's high resolution gravity data, for example, are possible at other solar system bodies.

Selected future exploration Concepts include:

Characterizing lce Thickness: RS experiments could provide stringent constraints on the thickness of ice and the characteristics of any sub-surface ocean at icy moons. NASA and ESA missions to Europa and Ganymede have such potential, and JUICE will utilize advanced radio instrumentation to explore the Jovian environment. Missions to other icy moons or small bodies could also exploit RS techniques.

Networks for Atmospheric Dynamics: Spacecraft-to-DSN radio occultations have unveiled structural details that have led to better understanding of atmospheric processes. Significant increases in temporal and spatial global coverage are possible using crosslinks among a network of small spacecraft orbiting a planet (Fig. 2), akin to radio occultation with constellations at Earth. Network science will also contribute to safe operations of future human and robotic Mars missions.

Networks for Interior Dynamics: Precision gravity experiments via spacecraft-to-spacecraft links can be applied to planetary targets for high-impact geophysical exploration of the interiors and monitoring of mass transport. Airless bodies such as Mercury are best suited for dual-spacecraft GRAIL-like production of high resolution gravitational fields, while GRACE-like resolved monitoring of time-variable gravity would considerably improve our understanding of the Martian climate.

Rotational State \& Tidal Deformations via Same Antenna Interferometry: Tracking two or more planetary landers from the same ground antenna enables high precision measurements as many common mode noise sources are suppressed. In turn, determination of the planetary rotational state and tidal deformations can be measured (Fig. 3)

Atmospheric Dynamics via Doppler Wind Experiments: Radio links from descending probes to proximity spacecraft or directly to ground stations provide information on dynamics relevant to atmospheric workings as well as planning the landing of future rovers. Dual-link Doppler measurements, one link between the probe and a spacecraft, and a second link between the probe and a ground station, allow derivation of two-dimensional wind vectors. Absorption on the probe's radio link can be used to infer concentrations of ammonia or sulfuric acid. Solar System Dynamics via Precision Ranging: ESA's BepiColombo Mercury mission will utilize coherent simultaneous $\mathrm{Ka}$ - and X-band Doppler links as well as the first ever precision ranging $(<20 \mathrm{~cm})$ at Ka-band. Advanced ranging instrumentation on-board the spacecraft and at the DSN will be utilized to investigate Solar System dynamics as well as tests of General Relativity.

Surface Properties from Scattering Studies: Bistatic radar in the uplink configuration provides higher SNR, as demonstrated by the New Horizons and LRO Mini-RF observations. Global spacecraft networks make surveying new planetary targets feasible and yield information on surface properties at spatial scales important to the safety of landers and rovers.

Enhanced Atmospheric \& Interior Science via Optical Links: With the advent of optical communications, laser links can augment radio links for atmospheric propagation science and provide precision opti-metrics to improve gravitational experiments and knowledge of Solar System ephemerides.

Enhanced Planetary Gravity via Atomic Clocks: Space borne atomic clocks would revolutionize spacecraft tracking methods. Future one-way uplinks could achieve accuracies comparable to traditional two-way coherent links. This could enable nearly continuous tracking using smaller ground antennas, opening a window for enhanced gravitational field measurements and improved models of planetary structures.

Solar System Dynamics Data Quality with Suppressed Antenna Mechanical Noise: With the most sensitive Doppler fractional frequency stability data to date $\approx 3 \times 10^{-15}$ (at $1000 \mathrm{~s}$ ), the leading noise was the unmodeled motion of the ground antenna's phase center. This intrinsic mechanical noise can be suppressed when two-way and (3-way) receive-only Doppler data from a smaller and stiffer antenna are suitably combined, further enhancing radio-metric observations for Solar System dynamics experiments.

\section{Selected Science-Enabling Technologies}

- Advanced precision ranging for Solar System dynamics and tests of General Relativity,

- Next generation ultra-stable oscillators,

- Chip-based atomic clocks

- Antenna mechanical noise reduction for precision Doppler gravity measurements,

- High power Ka-band transmitters for precision Doppler gravity measurements,

- Optical link science

- Uplink RS as a DSN service,

- RS spacecraft-to-spacecraft link instrumentation,

- Next generation Mars Cube One (MarCO) for RS

- Space-based assets and CubeSat networks, and

- Array communication architectures.

\section{References}

[1] less, L., et al., (2012) Science, 337, 457-459

[2] Asmar, S., et al., (2016), IEEE

Aerospace Conf.

[3] Gregnanin, M., (2012), Planetary and Space Science 74.1, 194-20

Acknowledgement:

This research was carried out in part at the Jet Propulsion Laboratory, California Institute of Technology, under a contract with the National Aeronautics and Space Administration. 